\title{
Ý ĐỊNH MUA VÀ SÃN SÀNG TRẢ GIÁ CAO CỦA NGƯờI TIÊU DÙNG ĐỐI Với THỰC PHẨM HŨ̉U CO: ỨNG DỤNG MÔ HİNH HỒI QUY LOGISTICS
}

\author{
NGUYẼ̃N NGỌC HIỀN \\ Khoa Quản trị kinh doanh, Trường Đại học Công nghiệp Thành phố Hồ Chí Minh \\ nguyenngochien.qn@iuh.edu.vn
}

Tóm tắt. Tình hình tiêu thụ thực phẩm hữu cơ đã tăng trong thời gian gần đây và đáp ứng được mối quan tâm của người tiêu dùng về các vấn đề liên quan đến sức khỏe, hạnh phúc và môi trường. Tuy nhiên, việc gia tăng tiêu thụ vẫn còn nhiều hạn chế so với tiềm năng của thị trường. Nghiên cứu này nhằm mục đích điều tra các yếu tố ảnh hưởng đến ý định mua và sẵn sàng trả giá cao của người tiêu dùng đối với thực phẩm hữu cơ. Với một mẫu nghiên cứu gồm 267 người tiêu dùng được khảo sát trực tiếp tại các hộ gia đình ở thành phố Hồ Chí Minh, các giả thuyết đã được kiểm định bằng mô hình hồi quy logistics. Kết quả xác nhận rằng, đặc điểm gia đình, thái độ, nhận thức an toàn và rào cản rủi ro là những yếu tố chính ảnh hưởng đáng kể đến ý định mua và sẵn sàng trả giá cao của người tiêu dùng đối với thực phẩm hữu cơ. Kết quả của nghiên cứu này cung cấp những hiểu biểt sâu sắc cho nhà quản lý và tiếp thị về các biến số chính thúc đẩy tiêu thụ thực phẩm hữu cơ trong nước.

Từ khóa. Thực phẩm hữu cơ, ý định mua hàng, sẵn sàng trả giá cao, hồi quy logistic.

\section{CONSUMERS' PURCHASE INTENTION AND WILLINGNESS TO PAY A PREMIUM FOR ORGANIC FOODS: APPLICATION OF THE BINARY LOGISTICS MODEL}

\begin{abstract}
The consumption of organic food has increased dramatically in recent years because consumers have gained concerns about health, well-being and the environment. However, the increase in consumption is still limited compared to the market potential. This study investigates the factors that influence consumers' purchase intention and willingness to pay a premium for organic foods. With a sample of 267 consumers surveyed directly in households in Ho Chi Minh City, the hypotheses have been tested by using a logistics regression model. The results confirm that family characteristics, attitude, safety perception and risk barrier are the main factors that significantly influence consumers' purchase intention and willingness to pay a premium for organic foods. The results of this study provide to regulators and marketers with insights of the key variables driving organic food consumption.
\end{abstract}

Keywords. Organic food, purchase intention, willingness to pay a premium, logistic regression.

\section{GIÖI THIÊUU}

Những năm gần đây đã chứng kiến sự gia tăng tiêu dùng thực phẩm được sản xuất thông qua phương pháp hữu cơ, vì nó được xem là thực phẩm thay thế lành mạnh và bền vững đối với môi trường [1-3]. Canh tác hữu cơ đã được áp dụng và mở rộng ở hơn 175 quốc gia [1]. Thị trường tiêu thụ thực phẩm hữu cơ toàn cầu ước đạt 97 tỷ USD và dự báo sẽ tiếp tục phát triển [4]. Tại Việt Nam, ngộ độc thực phẩm và tỷ lệ mắc bệnh do thực phẩm cao làm cho một bộ phận người tiêu dùng rất lo lắng về an toàn thực phẩm $[5,6]$. Thúc đẩy tiêu dùng thực phẩm bền vững là một trong những ưu tiên hàng đầu của quốc gia, nhằm giải quyết những lo ngại ngày càng tăng về các vấn đề môi trường, sức khỏe và an toàn thực phẩm $[7,8]$. Chính phủ đã thực hiện một số sáng kiến như Nghị định 109/2018/NĐ-CP ngày 29 tháng 8 năm 2018 về văn hóa hữu cơ và các tiêu chuẩn an toàn thực phẩm để thúc đẩy sự phát triển của ngành công nghiệp thực phẩm hữu cơ. Các nhà sản xuất và bán lẻ đã nỗ lực rất nhiều trong việc cung cấp các sản phẩm thực phẩm hữu cơ đa dạng (ví dụ, rau, ngũ cốc và thịt), cũng như mở rộng mạng lưới phân phối của họ [9]. Ngoài ra, Việt Nam là một thị trường mới nổi có dân số trên 95 triệu người, tong sản phẩm quốc nội tăng trưởng trung bình trên $6 \%$ trong thập kỷ qua (World bank, 2019), mức chi tiêu trung bình cho thực phẩm và đồ uống chiếm gần $50 \%$ thu nhập. Điều này cho thấy tiềm năng tiêu thụ thực phẩm hữu cơ tại Việt Nam còn rất lớn.

Hành vi tiêu dùng liên quan đến thực phẩm hữu cơ đã nhận được sự quan tâm lớn của các nhà nghiên cứu ở nhiều khía cạnh khác nhau như đặc điểm, sở thích, thái độ của người tiêu dùng, ý định mua, sẵn sàng mua và xu hướng tiêu thụ thực phẩm hữu cơ ở nhiều quốc gia khác nhau $[1,3,6,9,10]$. Bên cạnh đó, các nghiên 
cứu cũng thực hiện trên nhiều sản phẩm khác nhau về các chủ đề tương tự như sản phẩm tươi sống [11], thực phâm hữu cơ [10,12,13], dầu thực vật [14], và sữa hữu cơ [15]. Các nghiên cứu trước đây đã sử dụng lý kinh tế vi mô và kinh tế học hành vi để giải thích hành vi mua của người tiêu dùng và thử nghiệm trên nhiều sản phẩm khác nhau. Nhiều nghiên cứu đồng ý rằng, người tiêu dùng có thái độ tích cực đối với với thực phẩm hữu cơ và có ý định mua chúng. Tuy nhiên, phần lớn người tiêu dùng vẫn không sẵn sàng mua [16]. Điều này cho thấy, các nghiên cứu hiện tại vẫn chưa nghiên cứu đầy đủ trong mọi trường hợp và vẫn còn nhiều khoảng trống cần được nghiên cứu. Nhằm mục đích giải thích rõ hơn hành vi mua thực phẩm hữu cơ trong bối cảnh một quốc gia đang phát triển như Việt Nam. Mục tiêu của nghiên cứu này là xem xét ảnh hưởng của đặc điểm cá nhân, đặc điểm gia đình, thái độ, nhận thức về an toàn và rào cản rủi ro đến ý định mua và sẵn sàng trả giá cao cho thực phẩm hữu cơ của người tiêu dùng. Nghiên cứu này đóng góp vào tài liệu liên quan đến hành vi tiêu dùng thực phẩm hữu cơ theo những cách sau, thứ nhất nghiên cứu góp phần giải thích những đặc điểm bên ngoài và nhận thức bên trong của người tiêu dùng ảnh hưởng đến ý định mua và sẵn sàng trả giá cao, bổ sung kết quả kiểm định vào cuộc tranh luận liên quan đến hành vi tiêu dùng thực phẩm hữu cơ. Thứ hai, nghiên cứu này hỗ trợ các biên liên quan, đặc biệt là các doanh nghiệp sản xuất và kinh doanh thực phẩm hữu cơ, trong việc phát triển các chiến lược phù hợp để nâng cao hành vi mua thực phẩm hữu cơ của người tiêu dùng.

Trong nghiên cứu này, lý thuyết hành vi có kế hoạch (TBP) của Ajzen (1991) [17] làm khung khái niệm giải thích cho ý định mua và trả giá cao đối với thực phẩm hữu cơ. Lý thuyết này xác nhận rằng, hành vi cụ thể được thúc đẩy bởi ý định thực hiện hành vi của một người. Ý định phản ánh động cơ và có kế hoạch để thực hiện hành vi và được thúc đẩy bởi ba yếu tố nhận thức chính là thái độ, chuẩn chủ quan và nhận thức kiểm soát hành vi. TBP đã được chứng minh phù hợp để giải thích hành vi tiêu dùng xanh đối với nhiều loại sản phẩm khác nhau [10, 15].

Phần còn lại của nghiên cứu này được cấu trúc như dưới đây. Tổng quan tài liệu được đánh giá và xây dựng các giả thuyết nghiên cứu. Phương pháp nghiên cứu và kết quả được phân tích. Cuối cùng, các kết luận bao gồm ý nghĩa nghiên cứu và nghiên cứu trong tương lai được thảo luận.

\section{CƠ SỞ LÝ THUYÊT}

\subsection{Thực phẩm hữu cơ và ý định mua thực phẩm hữu co}

Ghali và Hamdi (2015) [18] xác định hệ thống sản xuất hữu cơ là các hoạt động canh tác không sử dụng phân bón, hóa chất và không ảnh hưởng đến môi trường. Các nhà nghiên cứu về tiếp thị coi nông nghiệp hữu cơ là quá trình sản xuất gắn với việc sử dụng hạn chế thuốc trừ sâu, thuốc diệt cỏ, ít hóa chất tồn dư để không ảnh hưởng đến môi trường và bảo vệ sức khỏe con người [19]. Thực phẩm hữu có thể được xác định là các sản phẩm được trồng mà không sử dụng thuốc trừ sâu, phân bón tổng hợp, bùn thải, sinh vật biến đổi gen hoặc bức xạ ion hóa, cũng như không có chất kháng sinh và chất kích thích tăng trưởng [20]. Đa số người tiêu dùng tin rằng thực phẩm hữu cơ thân thiện với môi trường, lành mạnh, sạch hơn, bổ dưỡng hơn, ngon hơn và an toàn hơn so với thực phẩm thông thường [21,22].

Nik Abdul Rashid (2009) [23] định nghĩa rằng ý định mua thực phẩm hữu cơ là khả năng ý chí của cá nhân trong việc thực hiện hành vi mua thực phẩm hữu cơ hơn thực phẩm khác trong việc cân nhắc mua sắm. Ramayah và cộng sự (2010) [24] cho rằng ý định mua thực phẩm hữu cơ là một trong những biểu hiện cụ thể của hành vi mua. Han và cộng sự (2009) [25] cho rằng ý định mua thực phẩm hữu cơ thường gắn với hành vi truyền miệng tốt về sản phẩm và sẽ có ý định trả nhiều tiền hơn cho sản phẩm hữu cơ.

\subsection{Sự sẵn sàng trả giá cao}

Sự sẵn sàng chi trả là một khái niệm đánh giá nhận thức của một người về giá trị và chất lượng của một thương hiệu, vì giá cao hơn phản ánh giá trị cao hơn và chất lượng tốt hơn [26]. Sự sẵn sàng trả giá cao của người tiêu dùng là cấu trúc kết quả, thu hút sự quan tâm của các nhà tiếp thị. Nó có nghĩa là sự sẵn sàng chi trả nhiều hơn cho một thương hiệu hoặc một sản phẩm cụ thể so với các thương hiệu hoặc sản phẩm thay thế tương đương [27]. Trong bối cảnh cạnh tranh đang ngày càng khốc liệt, các nhà tiếp thị bắt buộc phải hiểu được động cơ bên trong ảnh hưởng đến sự sẵn sàng trả tiền cho một sản phẩm thay vì phải lựa chọn các sản phẩm khác. Sự sẵn sàng trả giá cao của người tiêu dùng rõ ràng quan trọng đối với bất kỳ tổ chức kinh doanh nào, vì nó có thể dẫn đến lợi nhuận cao hơn và tạo ra lợi thế cạnh tranh bền vững cho tổ chức [28].

\subsection{Lý thuyết nền}

Theo lý thuyết về hành vi có kế hoạch (TPB), hành vi cụ thể của một cá nhân được xác định bởi ý định của họ để thực hiện hành vi đó [17]. TBP rất hữu ích và phổ biến trong việc dự đoán ý định cũng như hành vi 
của người tiêu dùng $[29,30]$. Dựa trên $\mathrm{TPB}$, nhiều mô hình nghiên cứu đã được phát triển để dự đoán ý định hành vi của người tiêu dùng trong bối cảnh tiêu dùng thực phẩm hữu cơ $[3,6,9,10]$. Thêm nữa, một ý định mua thực phẩm tốt hơn có thể dẫn đến mua nhiều thực phẩm hơn [31]. Tuy nhiên, có một khoảng cách giữa ý định của người tiêu dùng thực tế hành vi mua hàng trong các quyết định hàng ngày [32]. Biến số kinh tế được mô tả là yếu tố chính ảnh hưởng đến việc mua thực tế, chênh lệch giữa khả năng thanh toán và giá cả là rào cản đối với việc mua rau an toàn [33]. Do đó, cần có cái nhìn sâu sắc về mức độ sẵn sàng trả giá cao cho rau an toàn của người tiêu dùng. Trong nghiên cứu này, một mô hình nghiên cứu được phát triển cho các giả thuyết rằng, ý định mua và sẵn sàng trả giá cao của người tiêu dùng chịu ảnh hưởng bởi các yếu tố bên trong và các đặc điểm bên ngoài của người tiêu dùng.

\section{GIẢ THUYẾT VÀ MÔ HİNH NGHIÊNN CÚU}

Các yếu tố quan trọng nhất ảnh hưởng đến hành vi mua thực phẩm hữu cơ của người tiêu dùng gồm sự quan tâm đến môi trường, nhân khẩu học, lối sống và sự quan tâm đến sức khỏe, kiến thức, các yếu tố bên ngoài (chế biến, đóng gói, nhà cung cấp, hình ảnh cửu hàng...), nhận thức kiểm soát hành vi, chuẩn chủ quan và các yếu tố liên quan đến thuộc tính sản phẩm [34-38]. Ngoài ra, một số đặc điểm của cá nhân và gia đình được xem là yếu tố quan trọng ảnh hưởng đến hành vi của người tiêu dùng [36]. Dựa vào xem xét các tài liệu nghiên cứu về tiêu thụ thực phẩm hữu cơ. Nghiên cứu này xác định các yếu tố bao gồm, đặc điểm các nhân, đặc điểm gia đình, thái độ, nhận thức an toàn và rào cản rủi ro là những biến độc lập. Các biến phụ thuộc là ý định mua hàng và sự sẵn sàng trả giá cao cho thực phẩm hữu cơ.

\section{1. Đặc điểm cá nhân}

Nghiên cứu trước đây cho thấy rằng, hành vi của người tiêu dùng chịu sự ảnh hưởng bởi đặc điểm cá nhân của người tiêu dùng, hành vi có sự khác biệt đáng kể giữa những người tiêu dùng có đặc điểm khác nhau [39]. Nghiên cứu của D'Amico và cộng sự (2016) [40] cũng cho thấy rằng, đặc điểm cá nhân như giới tính, độ tuổi, trình độ giáo dục có ảnh hưởng đến hành vi mua thực tế của người tiêu dùng. Ngoài ra, một số nghiên cứu về hành vi tiêu dùng xanh của cho thấy rằng, đặc điểm của người tiêu dùng ảnh hưởng đến ý định mua hàng và sẵn sàng trả giá cao [35, 41]. Tuy nhiên, nghiên cứu của Zhang và cộng sự (2018) [38] trong bối cảnh rau an toàn tại Trung Quốc cho thấy rằng, đặc điểm cá nhân bao gồm giới tính, độ tuổi và trình độ giáo dục không ảnh hưởng đến ý định mua hàng và sẵn sàng trả giá cao của người tiêu dùng. Như vậy, có những kết quả khác biệt về ảnh hưởng của đặc điểm cá nhân đến ý định mua và sẵn sàng trả giá cao, tuy nhiên có thể đưa ra giả thuyết rằng:

Giả thuyết H1: Đặc điểm cá nhân của người tiêu dùng ảnh hưởng đến ý định mua thực phẩm hữu cơ (H1a) và sẵn sàng trả giá cao $(\mathrm{H} 1 \mathrm{~b})$.

\section{2. Đặc điểm gia đình}

Các kiểu gia đình khác nhau, tình trạng hôn nhân, sự hiện diện của trẻ em trong gia đình được xem là những yếu tố quan trọng ảnh hưởng tích cực đến thái độ cũng như hành vi mua thực phẩm hữu cơ của người tiêu dùng [36]. Các hộ gia đình có thu nhập cao hơn có nhiều khả năng hình thành thái độ tích cực và mua nhiều thực phẩm hữu cơ hơn [42]. Michaelidou và Hassan (2010) [43] nhận thấy rằng thu nhập hộ gia đình ảnh hưởng đến thái độ và ý định mua hàng của người tiêu dùng đối với sản phẩm hữu cơ. Ngoài ra, chi tiêu cho thực phẩm trong một gia đình thể hiện mức tiêu dùng và chất lượng tiêu dùng của người dân [44]. Nghiên cứu của Zhang và cộng sự (2018) [33] cho thấy rằng mức chi tiêu cho thực phẩm của một gia đình có ảnh hưởng tích cực đến ý định mua và sẵn sàng trả giá cao của người tiêu dùng. Ngoài ra, thu nhập của hộ gia đình cũng ảnh hưởng đển ý định mua và sự sẵn sàng trả giá cao [35]. Như vậy, có thể đưa ra giả thuyết:

Giả thuyết H2: Đặc điểm gia đình của người tiêu dùng ảnh hưởng đến ý định ý định mua thực phẩm hữu cơ $(\mathrm{H} 2 \mathrm{a})$ và sẵn sàng trả giá cao $(\mathrm{H} 2 \mathrm{~b})$.

\subsection{Thái độ}

Thái độ được xác định là xu hướng thuận lợi hoặc không thuận lợi của người tiêu dùng để thực hiện một hành vi cụ thể [21]. Thái độ đối với một hành vi càng thuận lợi, thì càng có nhiều khả năng cá nhân đó thực hiện một hành vi nhất định [3]. Thái độ đối với thực phẩm đã nổi lên như một yếu tố quan trọng ảnh hưởng đến ý định mua hàng của khách hàng [45]. Các nghiên cứu trước đây trong bối cảnh tiêu dùng thực phẩm hữu cơ đã chứng minh, thái độ là yếu tố quan trọng nhất ảnh hưởng đến ý định mua hàng $[45,46]$. Nhiều nghiên cứu cũng đã chứng minh thái độ các tác động mạnh mẽ đến ý định mua và sẵn sàng trả tiền $[30,33]$, và giả thuyết như sau: 
Giả thuyết H3: Thái độ của người tiêu dùng ảnh hưởng đến ý định mua thực phẩm hữu cơ $(\mathrm{H} 3 \mathrm{a})$ và sẵn sàng trả giá cao $(\mathrm{H} 3 \mathrm{~b})$.

\subsection{Nhận thức an toàn}

Do mức sống ngày càng được cải thiện và sự xuất hiện thường xuyên của các mối lo ngại về an toàn thực phẩm, đã dẫn đến mối quan tâm của người tiêu dùng về chất lượng và an toàn thực phẩm [47], [48]. An toàn có thể là một yếu tố góp phần vào giá trị thương hiệu khi tiếp thị sản phẩm thực phẩm cho người tiêu dùng [49]. Người tiêu dùng tin rằng thực phẩm an toàn, chẳng hạn như thực phẩm hữu cơ, thực phẩm xanh, sẽ ít hơn rủi ro hơn so với thực phẩm thông thường [50]. Cheng và cộng sự (2016) [51] thấy rằng người tiêu dùng mua rau thường xuyên chú ý đến an toàn thực phẩm chất lượng. Hơn nữa, Curlo (1999) [52] đã xác định rằng để nâng cao tính an toàn của sản phẩm, những sản phẩm đó phải được bán với giá cao hơn. Điều này sẽ dẫn đến người tiêu dùng phải trả tiền nhiều hơn cho rau an toàn. Nghiên cứu của Zhang và cộng sự (2018) [38] trong bối cảnh rau sạch ở trung quốc cũng cho thấy, nhận thức an toàn sẽ dẫn đến người tiêu dùng sẵn sàng trả giá cao hơn. Và giả thuyết như sau:

Giả thuyết H4: Nhận thức an toàn của người tiêu dùng ảnh hưởng đến ý định mua thực phẩm hữu cơ (H4a) và sẵn sàng trả giá cao $(\mathrm{H} 4 \mathrm{~b})$.

\subsection{Rào cản rủi ro}

Rào cản rủi ro phát sinh do nhận thức của người tiêu dùng về các mối đe dọa hoặc nguy cơ tiềm ẩn từ việc sử dụng một sản phẩm hoặc những cải tiến mới [2]. Các nghiên cứu trước đây đã cho thấy sự hoài nghi của người tiêu dùng về tính xác thực của các sản phẩm thực phẩm hữu cơ [53], chất lượng và chứng nhận [54]. Rủi ro liên quan đến việc mua thực phẩm hữu cơ được coi là lý do quan trọng chống lại việc mua và tiêu dùng thực phẩm hữu cơ [55]. Những rủi ro như vậy có thể liên quan đến ý định mua thực phẩm hữu cơ do người tiêu dùng nghi ngờ về các vấn đề liên quan đến tài chính, xã hội và lòng tin [56]. Ví dụ, người tiêu dùng có thể lo sợ rằng việc trả giá cao hơn cho thực phẩm được dán nhãn là sản xuất hữu cơo, nhưng có thể không có khác biệt đáng kể so với các thực phẩm thông thường [57]. Do đó, đồng tình với các quan điểm trong các nghiên cứu trước, rằng nhận thức rủi ro có tác động tiêu cực đến ý định mua và trả giá cao cho thực phẩm hữu cơ, và giả thuyết như sau:

Giả thuyết H5: Rào cản rủi ro ảnh hưởng đến tiêu cực đến ý định mua rau an toàn (H5a) và sẵn sàng trả giá cao (H5b).

\subsection{Mô hình nghiên cứu}

Dựa trên lý thuyết hành vi có kế hoạch của Ajzen (1991) [17] và các nghiên cứu trước. Mô hình nghiên cứu được đề xuất gồm 10 giả thuyết (Hình 1).

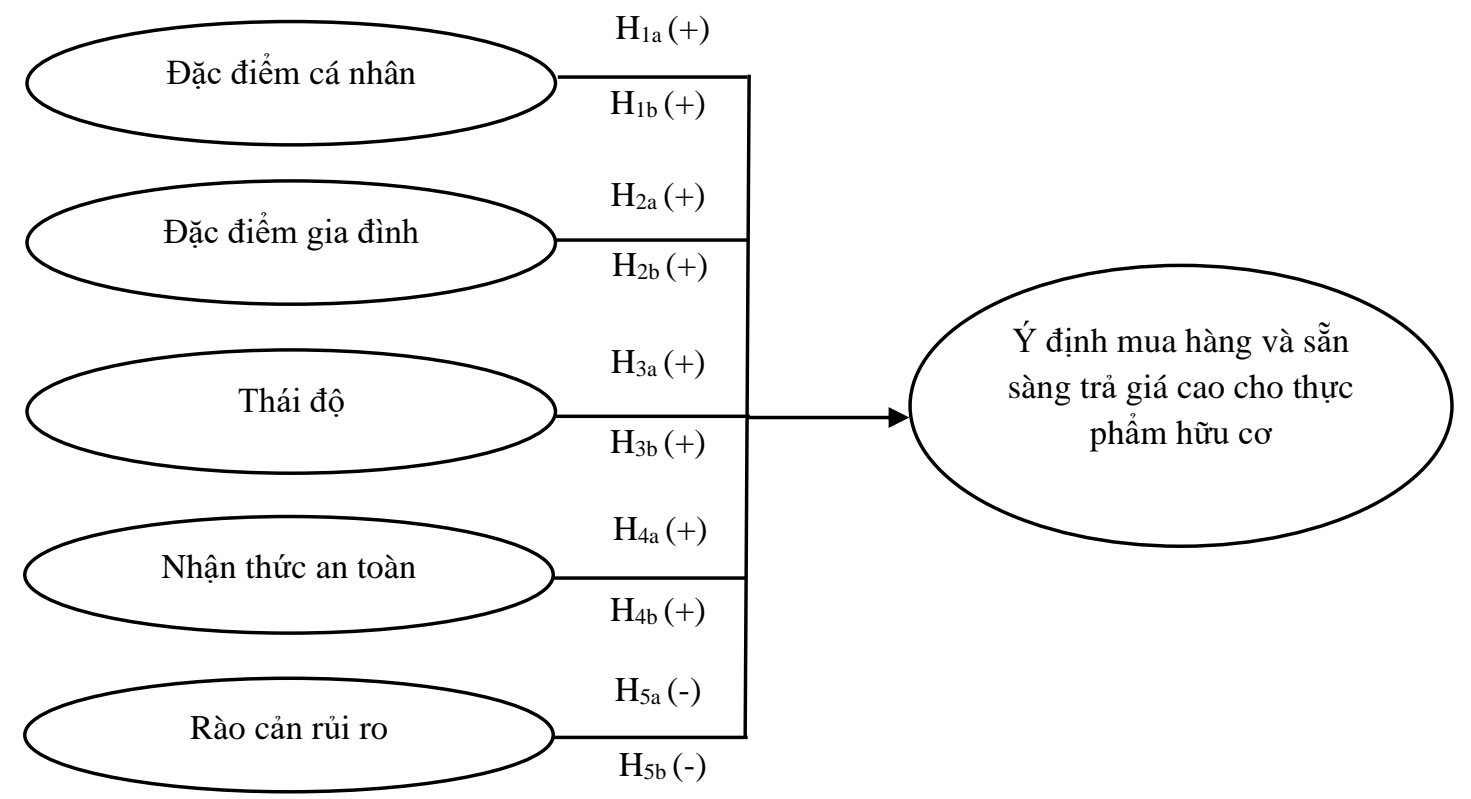

Hình 1: Mô hình nghiên cứu đề xuất

(Nguồn: Tác giả tổng hợp tù giả thuyết nghiên cưu) 


\section{PHƯƠNG PHÁP NGHIÊN CÚU}

\section{1. Đo lường}

Thang đo lường các khái niệm nghiên cứu được kế thừa từ thang đo lường của các nghiên cứu trước và được điều chỉnh để phù hợp với bối cảnh nghiên cứu này (Bảng 1 ). Các khái niệm đặc điểm cá nhân, đặc điểm gia đình, ý định mua và sẵn sàng trả giá cao được đo lường thông qua thang đo định tính. Các khái niệm thái độ, nhận thức an toàn và rào cản rủi ro được đo lường thông qua thang đo định lượng.

Một bảng câu hỏi khảo sát được thiết kế, đầu tiên một đoạn giới thiệu ngắn gọn về cuộc khảo sát, tiếp theo các câu hỏi gạn lọc về mức độ thường xuyên mua thực phẩm và có biết đến thực phẩm hữu cơ không, nhằm tập trung vào những người thường xuyên mua thực phẩm và có biết đến thực phẩm hữu cơ. Tiếp theo là các câu hỏi về các khái niệm trong mô hình nghiên cứu.

Một cuộc thảo luận nhóm được thực hiện vào tháng 5 năm 2020 nhằm điều chỉnh thang đo cho phù hợp với bối cảnh tại Việt Nam. Những người được chọn thường xuyên mua thực phẩm và đã từng mua thực phẩm hữu cơ tại thành phố Hồ Chí Minh. Tổng cộng có 08 người tham gia, những người tham gia thảo luận nhóm được yêu cầu đánh giá sự rõ ràng của bảng câu hỏi, đồng thời thảo luận các vấn đề liên quan đến thang đo như chi tiêu cho thực phẩm trung bình 1 tháng, thu nhập gia đình trung bình... Kết quả có sự điều chỉnh từ ngữ so với câu hỏi gốc để rõ nghĩa hơn, đồng thời xây dựng các thang đo thu nhập trung bình, chi tiêu trung bình cho việc mua thực phẩm để phù hợp với người dân địa phương (Bảng 1 thể hiện kết quả điều chỉnh).

Bảng 1: Cấu trúc, thang đo lường và nguồn tham khảo

\begin{tabular}{|c|c|c|c|}
\hline Biến quan sát & Ký hiệu & Giải thích biến & Thang do \\
\hline \multicolumn{4}{|c|}{ Đặc điểm cá nhân (Zhang và cộng sự, 2018) [38] } \\
\hline Giới tính & gioitinh & $\begin{array}{l}\text { Giới tính của người trả lời } \\
1=\text { nam; } 0=\text { nữ }\end{array}$ & Định tính \\
\hline Tuổi (năm) & tuoi & Tuổi của người trả lời & Định lượng \\
\hline Học vấn & hocvan & $\begin{array}{c}\text { Học vấn của người trả lời } \\
1=\text { trung học phổ thông trở lên; } 0=\text { khác }\end{array}$ & Định tính \\
\hline \multicolumn{4}{|c|}{ Đặc điểm gia đình (Cập nhật tù̀ Michaelidou và cộng sư, 2010) [43] } \\
\hline \multirow{3}{*}{$\begin{array}{l}\text { Thu nhập gia đình } \\
\text { trung bình } 1 \text { tháng }\end{array}$} & thunhap1 & $1=$ nhỏ hơn 10 triệu đồng; $0=$ =khác & \multirow{3}{*}{ Định tính } \\
\hline & thunhap2 & 1=10-20 triệu đồng; $0=$ khác & \\
\hline & thunhap3 & 1=lớn hơn 20 triệu đồng; $0=$ khác & \\
\hline \multirow{3}{*}{$\begin{array}{l}\text { Chi tiêu cho thực } \\
\text { phẩm trung bình } 1 \\
\text { tháng }\end{array}$} & chitieu 1 & $1=$ nhỏ hơn 3 triệu đồng; $0=$ khác & \multirow{3}{*}{ Định tính } \\
\hline & chitieu2 & 1=3-6 triệu đồng; $0=$ =khác & \\
\hline & chitieu3 & 1=lớn hơn 6 triệu đồng; $0=$ khác & \\
\hline Trẻ em & treem & 1=Có trẻ em dưới 10 tuổi trong gia đình; $0=$ không có & Định lượng \\
\hline \multicolumn{4}{|c|}{ Thái độ (Zhang và cộng sụ, 2018) [38] } \\
\hline Quen thuộc & quenthuoc & $\begin{array}{l}\text { Thang đo } 5 \text { điểm đo lường mức độ quen thuộc của } \\
\text { người tiêu dùng về thực phẩm hữu cơ, từ 1=Rất } \\
\text { không quen thuộc đến } 5=\text { Rất quen thuộc }\end{array}$ & Định lượng \\
\hline Sự khác biệt & khacbiet & $\begin{array}{c}\text { Thang đo } 5 \text { điểm đo lường nhận thức của người tiêu } \\
\text { dùng về sự khác biệt giữa thực phẩm hữu cơ và thực } \\
\text { phẩm khác, từ Không khác biệt=1 đến Khác biệt } \\
\text { lớn=5 }\end{array}$ & Định lượng \\
\hline \multicolumn{4}{|c|}{ Nhận thức an toàn (Cập nhật tì̀ Zhang và cộng sự, 2018) [38] } \\
\hline Mức độ an toàn & antoan & $\begin{array}{l}\text { Thang đo } 5 \text { điểm đo lường nhận thức của người tiêu } \\
\text { dùng về mức độ an toàn của thực phẩm, từ Rất } \\
\text { không tốt }=1 \text { đến Rất tốt }=5\end{array}$ & Định lượng \\
\hline Quan tâm an toàn & quantam & $\begin{array}{l}\text { Thang đo } 5 \text { điểm đo lường mức độ quan tâm của } \\
\text { người tiêu dùng về mức độ an toàn của thực phẩm, } \\
\text { từ Rất không quan tâm=1 đến Rất quan tâm=5 }\end{array}$ & Định lượng \\
\hline \multicolumn{4}{|c|}{$\begin{array}{l}\text { Rào cản rủi ro (ruiro) (Kushwah và công sur, 2019) [2] } \\
\text { Thang đo } 5 \text { điểm từ Rât không đồng ý=1 đến Rất đồng ý=5 }\end{array}$} \\
\hline Rủi ro 1 & ruiro1 & $\begin{array}{c}\text { Lo sợ thực phẩm hữu cơ trên thị trường không phải } \\
\text { là "hữu cơ" }\end{array}$ & Định lượng \\
\hline
\end{tabular}




\begin{tabular}{|l|c|c|c|}
\hline Rủi ro 2 & ruiro2 & $\begin{array}{c}\text { Lo sợ nhãn thực phẩm hữu cơ không xác thực (dễ bị } \\
\text { làm giả) }\end{array}$ & Định lượng \\
\hline Rủi ro 3 & ruiro3 & $\begin{array}{c}\text { Lo sợ nhà bán lẻ thực phẩm hữu cơ không đáng tin } \\
\text { cậy }\end{array}$ & Định lượng \\
\hline Biến phụ thuộc (Zhang và cộng sụ, 2018) [38] & $\begin{array}{c}\text { Có muốn mua thực phẩm hữu cơ không, Có=1; } \\
\text { Không=0 }\end{array}$ & Định tính \\
\hline Ý định mua & ydinh & $\begin{array}{c}\text { Có muốn trả giá cao cho thực phẩm hữu cơ so với } \\
\text { thực phẩm khác, Có=1; Không=0 }\end{array}$ & Định tính \\
\hline Sẵn sàng trả giá cao & giacao & \begin{tabular}{c} 
Côn \\
\hline
\end{tabular}
\end{tabular}

\subsection{Thu thập dữ liệu}

(Nguồn: Tác giả điều chỉnh dựa vào thang đo của nghiên cứu trước)

Một cuộc khảo sát chính thức được thực hiện từ ngày 01/06/2020 đến ngày 15/06/2020 tại thành phố Hồ Chí Minh. Khảo sát trực tiếp được thực hiện tại các hộ gia đình. Để tăng sự nhiệt tình những người tham gia được thông báo khi trả lời xong bảng câu hỏi sẽ được quà tặng. Người được hỏi ban đầu được yêu cầu trả lời các câu hỏi liên quan đến phần gạn lọc là người thường xuyên mua thực phẩm cho gia đình và biết đến thực phẩm hữu cơ, tiếp theo là trả lời các câu hỏi liên quan đến các khái niệm nghiên cứu. Số lượng bảng câu hỏi phát ra là 300, sau khi loại bỏ những bảng câu hỏi không phù hợp (trả lời không đầy đủ, có cùng câu trả lời cho tất cả câu hỏi), tổng cộng có 267 bảng câu hỏi hợp lệ được sử dụng để ước lượng và kiểm định mô hình nghiên cứu.

Mô hình hồi quy logistic được sử dụng để dự đoán kết quả của biến phụ thuộc phân loại dựa trên các biến độc lập [58]. Kỹ thuật này có thể sử dụng để phân tích hành vi mua của người tiêu dùng [38], [58] và sẵn sàng trả mức giá cao hơn [59], [60]. Trong nghiên cứu này, người tiêu dùng được hỏi có ý định mua thực phẩm hữu cơ không và có sẵn sàng trả giá cao không, như vậy các biến phụ thuộc trong mô hình này là biến giả (Thể hiện trong bảng 1). Mô hình hồi quy được thể hiện trong biểu thức 1.

$$
\mathrm{Y}_{i}=\ln \left(\frac{p_{i}}{1-p_{i}}\right)=\alpha+\sum_{j=1}^{n} \beta_{j} x_{i j}
$$

Trong đó, $\mathrm{Y}_{\mathrm{i}}$ là biến phụ thuộc, đại diện cho ý định mua thực phẩm hữu cơ của người tiêu dùng thứ i; $\mathrm{p}_{\mathrm{i}}$ là khả năng người thứ i mua thực phẩm hữu co; $\mathrm{x}_{\mathrm{j}}$ đại diện cho các biến độc lập trong mô hình; $\alpha$ là hằng số và $\beta_{\mathrm{j}}$ là hệ số của các biến độc lập được ước lượng. Tương tự, mô hình này cũng được sử dụng để ước lượng khả năng người tiêu dùng trả giá cao cho thực phẩm hữu cơ.

\section{KẾT QUẢ VÀ THẢO LUẬN}

\section{1. Đặc điểm mẫu thống kê}

Bảng 2 mô tả đặc điểm mẫu được khảo sát. Trong tổng số 267 người được khảo sát, những người có giới tính là nữ chiếm khoảng $64 \%$, độ tuổi trung bình là 32,5 tuổi, khoảng $70 \%$ có trình độ từ trung học phổ thông trở lên. Về đặc điểm gia đình, thu nhập lớn hơn 10 triệu đồng chiếm gần $80 \%$, hơn $60 \%$ số người được hỏi cho rằng chi tiêu thực phẩm trung bình 1 tháng lớn hơn 3 triệu đồng. Kết quả này chỉ ra rằng, những người được khảo sát là những người nằm trong độ tuổi lao động, có học vấn cao, có thu nhập ở mức cao hơn trung bình và có mức chi tiêu cho thực phẩm cũng tương đối cao. Ngoài ra, kết quả kiểm định cronbach's alpha cho thang đo đa biến "rủi ro", kết quả cho thấy rằng hệ số cronbach's alpha là 0,856 và hệ số tương quan biến-tổng lớn hơn 0,3 , như vậy thang đo rủi ro đạt độ tin cậy.

Bảng 2. Mô tả đặc điểm mẫu nghiên cứu $(\mathrm{n}=267)$

\begin{tabular}{|c|c|c|c|c|c|}
\hline Biến quan sát & Trung bình & Độ lệch chuẩn & Biến quan sát & Trung bình & Độ lệch chuẩn \\
\hline Giới tính & 0,36 & 0,49 & Sự khác biệt & 3,78 & 0,75 \\
\hline Tuổi & 32,5 & 8,52 & Mức độ an toàn & 2,16 & 0,53 \\
\hline Học vấn & 0,7 & 0,35 & $\begin{array}{c}\text { Quan tâm an } \\
\text { toàn }\end{array}$ & 3,89 & 0,78 \\
\hline Thu nhập gia đình/tháng & & & Rủi ro 1 & 3,78 & 0,68 \\
\hline <10 triệu đồng & 0,19 & 0,35 & Rủi ro 2 & 3,84 & 0,62 \\
\hline 10-20 triệu đồng & 0,34 & 0,42 & Rủi ro 3 & 3,65 & 0,71 \\
\hline >20 triệu đồng & 0,47 & 0,27 & Ý định mua & 0,72 & 0,30 \\
\hline Chi tiêu cho thực phẩn & & & Sẵn sàng trả & 0,68 & 0,36 \\
\hline
\end{tabular}



THỰC PHẨM HŨ̉U CO: ÚNG DỤNG MÔ HİNH HỒI QUY LOGISTICS

\begin{tabular}{|c|c|c|c|c|c|}
\hline$<3$ triệu đồng & 0,17 & 0,12 & giá cao & & \\
\hline 3-6 triệu đồng & 0,44 & 0,29 & Trẻ em & 0,72 & 0,46 \\
\hline$>6$ triệu đồng & 0,39 & 0,37 & Quen thuộc & 3,22 & 0,87 \\
\hline
\end{tabular}

\subsection{Các nhân tố ảnh hưởng đến ý định mua thực phẩm hữu co}

(Nguồn: Tác giả tổng hợp tù̀ dũ liệu khảo sát)

Bảng 3 trình bày kết quả ước lượng mổ hình hồi quy về ý định mua thực phẩm hữu cơ của người tiêu dùng. Kiểm định Omnibus cho thấy rằng $\operatorname{Sig} .=0,000<0,05$, như vậy có sự tương quan giữa biến độc lập và biến phụ thuộc trong mô hình với độ tin cậy trên $95 \%$. Hệ số $\mathrm{R}^{2}$ Nagelkerke=0,674, như vậy $67,4 \%$ sự thay đổi của ý định mua thực phẩm hữu cơ được giải thích bởi các biến trong mô hình [61].

Bảng 3. Kết quả hồi quy Binary logistic với biến phụ thuộc "ý định mua thực phẩm hữu cơ"

\begin{tabular}{|c|c|c|c|c|}
\hline Biến/khái niệm & Hệ số (B) & $\begin{array}{l}\text { Sai số chuẩn } \\
\text { (S.E) }\end{array}$ & $\begin{array}{l}\text { Mức ý nghĩa } \\
\text { (Sig.) }\end{array}$ & Giả thuyết \\
\hline \multicolumn{5}{|l|}{ Đặc điểm cá nhân } \\
\hline gioitinh & 0,028 & 0,481 & 0,320 & Bác bỏ \\
\hline tuoi & 0,157 & 0,241 & $0,036^{* *}$ & Chấp nhận \\
\hline hocvan & 0,062 & 0,238 & 0,214 & Bác bỏ \\
\hline \multicolumn{5}{|l|}{ Đặc điểm gia đình } \\
\hline thunhap2 & 0,487 & 0,354 & $0,000 * * *$ & Chấp nhận \\
\hline thunhap3 & 0,541 & 0,421 & $0,000 * * *$ & Chấp nhận \\
\hline chitieu2 & 0,451 & 0,238 & $0,000 * * *$ & Chấp nhận \\
\hline chitieu3 & 0,572 & 0,381 & $0,000 * * *$ & Chấp nhận \\
\hline treem & 0,335 & 0,471 & $0,000 * * *$ & Chấp nhận \\
\hline \multicolumn{5}{|l|}{ Thái đọ } \\
\hline quenthuoc & 0,217 & 0,431 & $0,025 * *$ & Chấp nhận \\
\hline khacbiet & 0,121 & 0,513 & 0,141 & Bác bỏ \\
\hline \multicolumn{5}{|l|}{ Nhận thức an toàn } \\
\hline antoan & 0,631 & 0,523 & $0,000 * * *$ & Chấp nhận \\
\hline quantam & 0,493 & 0,481 & $0,000 * * *$ & Chấp nhận \\
\hline Rào cản rủi ro & $-0,517$ & 0,579 & $0,000 * * *$ & Chấp nhận \\
\hline \multicolumn{5}{|c|}{ Số quan sát $=267 ; \mathrm{R}^{2}$ Nagelkerke $=0,674 ;$ Sig (kiểm định Omnibus) $=0,000$} \\
\hline
\end{tabular}

(Nguồn: Phân tích dũ liệu thu thập tì̀ phần mềm SPSS 24.0) Kết quả hồi quy cho thấy rằng, trong ba đặc điểm cá nhân gồm giới tính, độ tuổi và học vấn, thì chỉ có độ tuổi ảnh hưởng đến ý định mua thực phẩm hữu cơ. Kết quả này phù hợp với nghiên cứu của Zhang và cộng sự (2018) [38], Luu (2019) [35]. Các nghiên cứu này cũng cho thấy, các đặc điểm cá nhân ít ảnh hưởng đến ý định mua. Đều này khá hợp lý trong bối cảnh hiện nay, khi nhận thực về an toàn thực phẩm ngày càng tăng lên, vì vậy không phân biệt giới tính hay trình độ học vấn, người dân luôn quan tâm và có ý định mua thực phẩm hữu cơ. Về đặc điểm gia đình, các biến thu nhập, chi tiêu cho thực phẩm và có trẻ em trong gia đình đều có ảnh hưởng tích cực đến ý định mua thực phẩm hữu cơ. Kết quả này phù hợp một phần so với nghiên cứu của Zhang và cộng sự (2018) [38]. Đều này có nghĩa là xác suất mua thực phẩm hữu cơ sẽ cao hơn đối với những gia đình có thu nhập cao hơn, có nhu cầu mua thực phẩm hữu cơ cao hơn và xác suất mua cũng cao hơn đối với những gia đình có trẻ em nhỏ hơn 10 tuồi. Kết quả nghiên cứu cũng cho thấy, khách hàng càng quen thuộc với thực phẩm hữu cơ thì ý định mua của họ sẽ cao hơn, nhận thức an toàn cao hơn cũng ảnh hưởng tích cực đến ý định mua. Ngoài ra, rào cản rủi ro là yếu tố quan trọng ảnh hưởng tiêu cực đến xác suất mua thực phẩm hữu cơ của người tiêu dùng. Đều này cho thấy rằng, người tiêu dùng vẫn chưa tin tưởng các sản phẩm hữu cơ trên thị trường và đều này ảnh hưởng đến ý định mua của họ.

\subsection{Các nhân tố ảnh hưởng đến ý định sã̃n sàng trả giá cao cho thực phẩm hữu cơ}

Bảng 4 trình bày kết quả ước lượng mô hình hồi quy về ý định trả giá cao cho thực phẩm hữu cơ của người tiêu dùng. Kiểm định Omnibus cho thấy rằng $\mathrm{Sig} .=0,000<0,05$, như vậy có sự tương quan giữa biến độc lập và biến phụ thuộc trong mô hình với độ tin cậy trên $95 \%$. Hệ số $\mathrm{R}^{2}$ Nagelkerke $=0,720$, như vậy $72 \%$ sự thay đổi của ý định trả giá cao cho thực phẩm hữu cơ được giải thích bởi các biến trong mô hình. 
Bảng 4. Kết quả hồi quy Binary logistic với biến phụ thuộc "ý định trả giá cao cho thực phẩm hữu cơ"

\begin{tabular}{|c|c|c|c|c|}
\hline Biến/khái niệm & Hệ số (B) & $\begin{array}{l}\text { Sai số chuẩn } \\
\text { (S.E) }\end{array}$ & $\begin{array}{l}\text { Mức ý nghĩa } \\
\text { (Sig.) }\end{array}$ & Giả thuyết \\
\hline \multicolumn{5}{|l|}{ Đặc điểm cá nhân } \\
\hline gioitinh & 0,231 & 0,512 & 0,027 ** & Chấp nhận \\
\hline tuoi & 0,072 & 0,501 & 0,219 & Bác bỏ \\
\hline hocvan & 0,201 & 0,410 & $0,039 * *$ & Chấp nhận \\
\hline \multicolumn{5}{|l|}{ Đặc điểm gia đình } \\
\hline thunhap2 & 0,701 & 0,610 & $0,000 * * *$ & Chấp nhận \\
\hline thunhap3 & 0,571 & 0,310 & $0,000 * * *$ & Chấp nhận \\
\hline chitieu2 & 0,310 & 0,391 & $0,000 * * *$ & Chấp nhận \\
\hline chitieu3 & 0,297 & 0,261 & $0,000 * * *$ & Chấp nhận \\
\hline Treem & 0,151 & 0,430 & $0,042 * *$ & Chấp nhận \\
\hline \multicolumn{5}{|l|}{ Thái độ } \\
\hline quenthuoc & 0,019 & 0,516 & 0,421 & Bác bỏ \\
\hline khacbiet & 0,217 & 0,281 & $0,023^{* *}$ & Chấp nhận \\
\hline \multicolumn{5}{|l|}{ Nhận thức an toàn } \\
\hline antoan & 0,319 & 0,410 & $0,000 * * *$ & Chấp nhận \\
\hline quantam & 0,510 & 0,610 & $0,000 * * *$ & Chấp nhận \\
\hline Rào cản rủi ro & $-0,319$ & 0,424 & $0,000 * * *$ & Chấp nhận \\
\hline \multicolumn{5}{|c|}{ Số quan sát $=267 ; \mathrm{R}^{2}$ Nagelkerke $=0,720 ; \mathrm{Sig}$ (kiểm định Omnibus) $=0,000$} \\
\hline
\end{tabular}

(Nguồn: Phân tích dũ liệu thu thập tù phần mềm SPSS 24.0) Về đặc điểm cá nhân, học vấn có ảnh hưởng tích cực đến ý định trả giá cao, các đặc điểm giới tính và tuổi của người trả lời không có ảnh hưởng. Kết quả này trái ngược với kết quả nghiên cứu của Zhang và cộng sự (2018) [38] nhưng phù hợp với kết quả nghiên cứu của Luu (2019) [35]. Về đặc điểm gia đình, các biến thu nhập của hộ gia đình, chi tiêu cho thực phẩm và trẻ em đều có ảnh hưởng tích cực đến ý định trả giá cao để mua thực phẩm hữu cơ, kết quả cũng trái ngược với kết quả nghiên cứu của Zhang và cộng sự (2018) [38], đều này có thể giải thích do bối cảnh nghiên và đối tượng nghiên cứu khác nhau, nghiên cứu này thực hiện trên nhiều đối tượng có đặc điểm khác nhau về thu nhập và chi tiêu, vì vậy kết quả có sự biến thiên giữa các đối tượng. Kết quả cũng cho thấy, nhận thức sự khác biệt giữa thực phẩm hữu cơ và thực phẩm khác, nhận thức về an toàn của thực phẩm và sự quan tâm đến thực phẩm hữu cơ cũng ảnh hưởng tích cực đến ý định trả giá cao cho thực phẩm hữu cơ. Tuy nhiên, kết quả cũng cho thấy, người tiêu dùng lo ngại về tính chính xác của thực phẩm hữu cơ trên thị trường, đều này dẫn đến giảm ý định trả giá cao cho sản phẩm.

\section{KẾT LUẬN}

Chủ đề về tiêu dùng thực phẩm hữu cơ đã nhận được sự quan tâm ngày càng tăng cả trong học thuật và thực tiễn, nhằm giải quyết những lo ngại ngày càng tăng về các vấn đề môi trường, sức khỏe và an toàn thực phẩm. Dựa trên bối cảnh thực phẩm hữu cơ tại Việt Nam, nghiên cứu này đã phân tích đặc điểm, nhận thức của người tiêu dùng, ý định mua hàng và sẵn sàng trả giá cao. Đóng góp của nghiên cứu này là phát triển một mô hình mua thực phẩm hữu cơ của người tiêu dùng, nhằm xem xét các yếu tố làm tăng ý định mua và sẵn sàng trả giá cao trong bối cảnh thực phẩm hữu cơ. Dựa trên 267 bảng câu hỏi hợp lệ được khảo sát từ người tiêu dùng, mô hình đã được kiểm định bằng phương pháp hồi quy logistic. Kết quả của nghiên cứu này cung cấp những gợi ý thú vị cho các chiến lược kinh doanh và tiếp theo dựa trên nhận thức của người tiêu dùng trong bối cảnh thực phẩm hữu cơ tại Việt Nam.

Thứ nhất, những gia đình có thu nhập càng cao, chi tiêu cho thực phẩm càng nhiều thì càng có ý định mua thực phẩm hữu cơ. Điều này cho thấy rằng, nhu cầu thực phẩm hữu cơ tại Việt Nam ngày càng tăng lên dựa vào mức thu nhập của người dân ngày càng được cải thiện. Thêm nữa, những gia đình có trẻ em sẽ có ý định mua cao hơn những gia đình không có trẻ em. Vì vậy, các doanh nghiệp kinh doanh thực phẩm hữu cơ nên có phân tích hành vi mua của những người có thu nhập cao và những gia đình có trẻ em để xây dựng chiến lược marketing phù hợp hơn. Thêm nữa, mức độ nhận biết về thực phẩm hữu và nhận thức về thực phẩm an toàn cũng ảnh hưởng đến ý định mua của người tiêu dùng. Đều này đòi hỏi các doanh nghiệp cần 


\section{Ý ĐỊNH MUA VÀ SÃN SÀNG TRẢ GIÁ CAO CỦA NGƯỜI TIÊU DÙNG ĐỐI VỚI THỰC PHÂMM HỨU CƠ: ÚNGG DỤNG MÔ HİNH HỒI QUY LOGISTICS}

đầu tư hơn nữa trong chiến lược tiếp thị để nâng cao nhận biết của khách hàng, đồng thời cũng thông tin nhiều hơn để khách hàng nhận thức được về vấn đề an toàn khi sử dụng thực phẩm. Đều này sẽ góp phần nâng cao ý định sử dụng thực phẩm hữu cơ của người tiêu dùng. Ngoài ra, nghiên cứu cũng cho thấy, người tiêu dùng cũng chưa tin tưởng vào thực phẩm hữu cơ, điều này làm giảm ý định mua của người tiêu dùng. Vì vậy, việc cung cấp đầy đủ thông tin thông qua các phương tiện thông tin đại chúng khác nhau, thực hiện các phương pháp xác thực và giám sát nghiệm ngặt quy trình đảm bảo chất lượng nhằm mục đích thuyết phục người tiêu dùng tin tưởng và nâng cao ý định mua thực phẩm hữu cơ.

Thứ hai, về ý định trả giá cao của người tiêu dùng. Người tiêu dùng có trình độ cao, có thu nhập càng cao, càng mua nhiều thực phẩm và có trẻ em sẽ có xu hướng chấp nhận trả giá cao cho thực phẩm hữu cơ. Đều này gợi ý cho những nhà tiếp thị lựa chọn thị trường mục tiêu phù hợp với thị trường thực phẩm hữu cơ. Mức độ phân biệt so với thực phẩm khác và nhận thức an toàn của người tiêu dùng cũng ảnh hưởng đến ý định sẵn sàng trả giá cao. Vì vậy, việc quảng bá các sản phẩm hữu cơ giữa các doanh nghiệp nông nghiệp cần được đầu tư một cách hợp lý liên quan đến các đặc tính của sản phẩm và các vấn đề sức khỏe, những điều này sẽ giúp người tiêu dùng phân biệt và nhận thức được những ưu điểm của thực phẩm hữu cơ, để từ đó chấp nhận trả giá cao để nhận được giá trị cao hơn. Tuy nhiên, nếu người tiêu dùng không tin tưởng vào thực phẩm hữu cơ, nó sẽ làm giảm ý định trả giá cao. Vì vậy, các nhà tiếp thị cũng cần đưa thông tin về phương pháp sản xuất, lợi ích môi trường, ngoài ra cũng cần tối ưu chi phí để đảm bảo người tiêu dùng tin tưởng và chấp nhận trả giá cao cho thực phẩm hữu cơ.

Như vậy, nghiên cứu này đã xây dựng hai mô hình logistic để kiểm định các yếu tố ảnh hưởng đến ý định mua và sẵn sàng trả giá cao cho thực phẩm hữu cơ. Nghiên cứu đã đóng góp những gợi ý quan trọng đối với các doanh nghiệp sản xuất và tiếp thị thực phẩm hữu cơ, góp phần tăng cường tiêu thụ thực phẩm hữu cơ trong bối cảnh người tiêu dùng tại Việt Nam.

\section{TÀI LIỆU THAM KHẢO}

[1] A. Tandon, A. Dhir, P. Kaur, S. Kushwah, and J. Salo, "Behavioral reasoning perspectives on organic food purchase," Appetite, vol. 154, p. 104786, 2020, doi: 10.1016/j.appet.2020.104786.

[2] S. Kushwah, A. Dhir, and M. Sagar, "Understanding consumer resistance to the consumption of organic food. A study of ethical consumption, purchasing, and choice behaviour," Food Qual. Prefer., vol. 77, pp. 1-14, 2019, doi: 10.1016/j.foodqual.2019.04.003.

[3] K. Madan and R. Yadav, "Behavioural intention to adopt mobile wallet: a developing country perspective," J. Indian Bus. Res., 2016.

[4] H. Willer and J. Lernoud, The world of organic agriculture. Statistics and emerging trends 2019. Research Institute of Organic Agriculture FiBL and IFOAM Organics International, 2019.

[5] H. M. Ngo, R. Liu, M. Moritaka, and S. Fukuda, "Urban consumer trust in safe vegetables in Vietnam: The role of brand trust and the impact of consumer worry about vegetable safety," Food Control, vol. 108, no. March 2019, p. 106856, 2020, doi: 10.1016/j.foodcont.2019.106856.

[6] J. Ehlert and N. K. Faltmann, Food Anxiety in Globalising Vietnam. Springer Nature, 2019.

[7] J. I. J. C. De Koning, M. R. M. Crul, R. Wever, and J. C. Brezet, "Sustainable consumption in Vietnam: an explorative study among the urban middle class," Int. J. Consum. Stud., vol. 39, no. 6, pp. 608-618, 2015.

[8] T. T. Truong, M. H. T. Yap, and E. M. Ineson, "Potential Vietnamese consumers' perceptions of organic foods," Br. Food J., 2012.

[9] L. Van Huy, M. T. T. Chi, A. Lobo, N. Nguyen, and P. H. Long, "Effective segmentation of organic food consumers in Vietnam using food-related lifestyles," Sustain., vol. 11, no. 5, pp. 1-16, 2019, doi: $10.3390 /$ su 11051237.

[10] T. H. Pham, T. N. Nguyen, T. T. H. Phan, and N. T. Nguyen, "Evaluating the purchase behaviour of organic food by young consumers in an emerging market economy," J. Strateg. Mark., vol. 27, no. 6, pp. 540-556, 2019, doi: 10.1080/0965254X.2018.1447984.

[11] S. Sillani and F. Nassivera, "Consumer behavior in choice of minimally processed vegetables and implications for marketing strategies," Trends Food Sci. Technol., vol. 46, no. 2, pp. 339-345, 2015.

[12] M. McEachern, C. Seaman, S. Padel, and C. Foster, "Exploring the gap between attitudes and behaviour," Br. food J., 2005.

[13] C.-C. Teng and Y.-M. Wang, "Decisional factors driving organic food consumption," Br. Food J., 2015.

[14] N. A. Nielsen, T. Bech-Larsen, and K. G. Grunert, "Consumer purchase motives and product perceptions: a laddering study on vegetable oil in three countries," Food Qual. Prefer., vol. 9, no. 6, pp. 455-466, 1998.

[15] V. Carfora et al., "Explaining consumer purchase behavior for organic milk: Including trust and green self- 
identity within the theory of planned behavior," Food Qual. Prefer., vol. 76, no. March, pp. 1-9, 2019, doi: 10.1016/j.foodqual.2019.03.006.

[16] A. Markosyan, J. J. McCluskey, and T. I. Wahl, "Consumer response to information about a functional food product: apples enriched with antioxidants,” Can. J. Agric. Econ. Can. d'agroeconomie, vol. 57, no. 3, pp. 325-341, 2009.

[17] I. Ajzen, "The theory of planned behaviour. Organizational Behaviour and Human Decision Processes, 50 (2), 179-211," View, 1991.

[18] Z. Ghali and R. Hamdi, "The Purchase and Consumption Motivations of an Organic Product by the Tunisian Consumer: an application on the Prickly Pear Seed Oil (PPS)," J. North African Res. Bus., vol. 2015, pp. 116, 2015, doi: 10.5171/2015.220428.

[19] B. Chekima, A. I. Oswald, S. A. W. S. K. Wafa, and K. Chekima, "Narrowing the gap: Factors driving organic food consumption," J. Clean. Prod., vol. 166, pp. 1438-1447, 2017, doi: 10.1016/j.jclepro.2017.08.086.

[20] M. J. Dahm, A. V. Samonte, and A. R. Shows, "Organic foods: Do eco-friendly attitudes predict ecofriendly behaviors?," J. Am. Coll. Heal., vol. 58, no. 3, pp. 195-202, 2009, doi: 10.1080/07448480903295292.

[21] S. Smith and A. Paladino, "Eating clean and green? Investigating consumer motivations towards the purchase of organic food," Australas. Mark. J., vol. 18, no. 2, pp. 93-104, 2010.

[22] H. V. Nguyen, N. Nguyen, B. K. Nguyen, A. Lobo, and P. A. Vu, "Organic food purchases in an emerging market: The influence of consumers' personal factors and green marketing practices of food stores," Int. $J$. Environ. Res. Public Health, vol. 16, no. 6, 2019, doi: 10.3390/ijerph16061037.

[23] N. Rashid, "Awareness of eco-label in Malaysia's green marketing initiative," Int. J. Bus. Manag., vol. 4, no. 8, pp. 132-141, 2009.

[24] T. Ramayah, J. W. C. Lee, and O. Mohamad, "Green product purchase intention: Some insights from a developing country,” Resour. Conserv. Recycl., vol. 54, no. 12, pp. 1419-1427, 2010.

[25] H. Han, L.-T. J. Hsu, and J.-S. Lee, "Empirical investigation of the roles of attitudes toward green behaviors, overall image, gender, and age in hotel customers' eco-friendly decision-making process," Int. J. Hosp. Manag., vol. 28, no. 4, pp. 519-528, 2009.

[26] N. S. Davcik and P. Sharma, "Impact of product differentiation, marketing investments and brand equity on pricing strategies," Eur. J. Mark., 2015.

[27] R. G. Netemeyer et al., "Developing and validating measures of facets of customer-based brand equity," $J$. Bus. Res., vol. 57, no. 2, pp. 209-224, 2004.

[28] R. Casidy and W. Wymer, "A risk worth taking: Perceived risk as moderator of satisfaction, loyalty, and willingness-to-pay premium price," J. Retail. Consum. Serv., vol. 32, pp. 189-197, 2016.

[29] D. Zhikun and N. Fungfai, "Knowledge sharing among architects in a project design team," Chinese Manag. Stud., 2009.

[30] Y. Kim and H. Han, "Intention to pay conventional-hotel prices at a green hotel-a modification of the theory of planned behavior," J. Sustain. Tour., vol. 18, no. 8, pp. 997-1014, 2010.

[31] Q. Zhu, Y. Li, Y. Geng, and Y. Qi, "Green food consumption intention, behaviors and influencing factors among Chinese consumers," Food Qual. Prefer., vol. 28, no. 1, pp. 279-286, 2013.

[32] I. Schäufele and U. Hamm, "Organic wine purchase behaviour in Germany: Exploring the attitudebehaviour-gap with data from a household panel," Food Qual. Prefer., vol. 63, pp. 1-11, 2018.

[33] C. Y. Zhang, B. Yu, J. W. Wang, and Y. M. Wei, "Impact factors of household energy-saving behavior: An empirical study of Shandong Province in China," Journal of Cleaner Production, vol. 185. pp. 285-298, 2018, doi: 10.1016/j.jclepro.2018.02.303.

[34] A. Singh and P. Verma, "Factors influencing Indian consumers' actual buying behaviour towards organic food products," J. Clean. Prod., vol. 167, pp. 473-483, 2017, doi: 10.1016/j.jclepro.2017.08.106.

[35] D. T. Luu, "Willingness to pay and actual purchase decision for organic agriculture products in Vietnam," Econ. J. Emerg. Mark., vol. 11, no. 2, pp. 123-134, 2019, doi: 10.20885/ejem.vol11.iss2.art1.

[36] L. H. Essoussi and M. Zahaf, "Decision making process of community organic food consumers: an exploratory study," J. Consum. Mark., 2008.

[37] A. Tandon, A. Dhir, P. Kaur, S. Kushwah, and J. Salo, "Behavioral reasoning perspectives on organic food purchase," Appetite, vol. 154, p. 104786, 2020.

[38] B. Zhang, Z. Fu, J. Huang, J. Wang, S. Xu, and L. Zhang, "Consumers' perceptions, purchase intention, and willingness to pay a premium price for safe vegetables: A case study of Beijing, China," J. Clean. Prod., vol. 197, pp. 1498-1507, 2018, doi: 10.1016/j.jclepro.2018.06.273.

[39] R. S. Hughner, P. McDonagh, A. Prothero, C. J. Shultz, and J. Stanton, "Who are organic food consumers? A compilation and review of why people purchase organic food," J. Consum. Behav. An Int. Res. Rev., vol. 


\section{Ý ĐỊNH MUA VÀ SÃN SÀNG TRẢ GIÁ CAO CỦA NGƯỜI TIÊU DÙNG ĐỐI VỞI THỰC PHÂMM HỨU CƠ: ÚNGG DỤNG MÔ HINNH HỒI QUY LOGISTICS}

6, no. 2-3, pp. 94-110, 2007.

[40] G. di Vita, M. D'amico, A. Lombardi, and B. Pecorino, "Evaluating trends of low sodium content in food: the willingness to pay for salt-reduced bread, a case study.," Agric. Econ. Rev., vol. 17, no. 2, pp. 82-99, 2016.

[41] P. Xu, Y. Zeng, Q. Fong, T. Lone, and Y. Liu, “Chinese consumers' willingness to pay for green-and ecolabeled seafood," Food Control, vol. 28, no. 1, pp. 74-82, 2012.

[42] K. P. Aryal, P. Chaudhary, S. Pandit, and G. Sharma, "Consumers' willingness to pay for organic products: a case from Kathmandu valley," J. Agric. Environ., vol. 10, pp. 15-26, 2009.

[43] N. Michaelidou and L. M. Hassan, "Modeling the factors affecting rural consumers' purchase of organic and free-range produce: A case study of consumers' from the Island of Arran in Scotland, UK," Food Policy, vol. 35, no. 2, pp. 130-139, 2010, doi: 10.1016/j.foodpol.2009.10.001.

[44] F. D. Davis, "Perceived usefulness, perceived ease of use, and user acceptance of information technology," MIS Q., pp. 319-340, 1989.

[45] R. Yadav and G. S. Pathak, "Young consumers' intention towards buying green products in a developing nation: Extending the theory of planned behavior," J. Clean. Prod., vol. 135, pp. 732-739, 2016, doi: 10.1016/j.jclepro.2016.06.120.

[46] M. A. Ashraf, M. H. R. Joarder, and S. R. A. Ratan, “Consumers' anti-consumption behavior toward organic food purchase: an analysis using SEM,” Br. Food J., 2019.

[47] A. Bearth, M.-E. Cousin, and M. Siegrist, "Poultry consumers' behaviour, risk perception and knowledge related to campylobacteriosis and domestic food safety," Food Control, vol. 44, pp. 166-176, 2014.

[48] W. M. S. Yee, R. M. W. Yeung, and J. Morris, "Food safety: building consumer trust in livestock farmers for potential purchase behaviour," Br. Food J., 2005.

[49] Z. Wang, Y. Mao, and F. Gale, "Chinese consumer demand for food safety attributes in milk products," Food Policy, vol. 33, no. 1, pp. 27-36, 2008.

[50] X. Yu, Z. Gao, and Y. Zeng, "Willingness to pay for the 'Green Food' in China," Food Policy, vol. 45, pp. 80-87, 2014.

[51] L. Cheng et al., "Consumers' behaviors and concerns on fresh vegetable purchase and safety in Beijing urban areas, China," Food Control, vol. 63, pp. 101-109, 2016.

[52] E. Curlo, "Marketing strategy, product safety, and ethical factors in consumer choice," J. Bus. Ethics, vol. 21, no. 1, pp. 37-48, 1999.

[53] M. Watanabe et al., "Global structures of automotive interiors revealed by algorithms of the visual brain," Des. Stud., vol. 62, pp. 100-128, 2019, doi: 10.1016/j.destud.2018.10.004.

[54] J. Wojciechowska-Solis and A. Soroka, "Motives and barriers of organic food demand among Polish consumers," Br. Food J., 2017.

[55] S. Kushwah, A. Dhir, and M. Sagar, "Ethical consumption intentions and choice behavior towards organic food. Moderation role of buying and environmental concerns," J. Clean. Prod., vol. 236, p. 117519, 2019.

[56] J. Chen, A. Lobo, and N. Rajendran, "Drivers of organic food purchase intentions in mainland C hinaevaluating potential customers' attitudes, demographics and segmentation," Int. J. Consum. Stud., vol. 38, no. 4, pp. 346-356, 2014.

[57] F. J. Torres-Ruiz, M. Vega-Zamora, and M. Parras-Rosa, "Sustainable consumption: Proposal of a multistage model to analyse consumer behaviour for organic foods," Bus. Strateg. Environ., vol. 27, no. 4, pp. 588-602, 2018.

[58] H. Guo, B. Li, Y. Hou, S. Lu, and B. Nan, "Rural households' willingness to participate in the Grain for Green program again: A case study of Zhungeer, China," For. Policy Econ., vol. 44, pp. 42-49, 2014.

[59] S. Rousseau and L. Vranken, "Green market expansion by reducing information asymmetries: Evidence for labeled organic food products," Food Policy, vol. 40, pp. 31-43, 2013.

[60] K. Zhou and S. Yang, "Understanding household energy consumption behavior: The contribution of energy big data analytics," Renewable and Sustainable Energy Reviews, vol. 56. pp. 810-819, 2016, doi: 10.1016/j.rser.2015.12.001.

[61] A. Agresti, "Building and applying logistic regression models," Categ. Data Anal., pp. 211-266, 2007.

Ngày nhận bài: 01/10/2020

Ngày chấp nhận đăng: 04/02/2021 